\title{
Specifying and Model Checking Workflows of Single Page Applications with TLA ${ }^{+}$
}

\author{
Gefei Zhang \\ Hochschule für Technik und Wirtschaft Berlin \\ gefei.zhang@htw-berlin.de
}

\begin{abstract}
Single Page Applications (SPAs) are different than hypertextbased web applications in that their workflow is not defined by explicit links, but rather implicitly by changes of their widgets' states. The workflow may hence be hard to track. We present an approach to specifying and model checking SPAs with $\mathrm{TLA}^{+}$. Our approach makes it easier to document and to track the workflow of SPAs and to find potential design flaws.
\end{abstract}

\section{Introduction}

Single Page Applications (SPAs) are web applications that "interact with the user by dynamically rewriting the current page rather than loading entire new pages from a server" [16]. Compared with hypertext-based web applications, SPAs do not have an explicit navigation structure. Instead, their workflow is controlled implicitly by the states of their control elements (widgets). For example, widgets may appear or disappear to provide different information to the user, the information provided by a widget may change over time, and widgets may be disabled to disallow the user to perform a certain action.

While formal specification and validation of the navigation structure has been proved valuable in the area of hypertext-based web applications, see [1], analysis of workflows of SPAs has been little investigated. In this paper, we present an approach to specifying and model checking [4] the workflow of SPAs with TLA ${ }^{+}$.

$\mathrm{TLA}^{+}[12]$ is a formal specification language based on Temporal Logic of Actions. A TLA ${ }^{+}$module consists of variables and actions. The valuation of the variables determines the current state of the system, the actions define possible transitions between the states. TLA ${ }^{+}$also provides tools to model check or to theorem proof [6] the specification. Of the two methods, model checking is easier to use since its fully automatic. This paper focuses on model checking only.

We use variables to model user observable properties of widgets and actions to model user actions. The mapping is straight forward and thus practice friendly. Our approach helps to understand the requirements for the SPA better, and model checking helps to find potential design errors.

The reminder of this paper is organized as follows: In the following Sect. 2 we give a brief introduction to TLA ${ }^{+}$. In Sect. 3 we illustrate our approach by a simple example. We show examples of model checking the specification in Sect. 4 . 
Related work is discussed in Sect. 5, before in Sect. 6 we draw conclusions and sketch some future work.

\section{$2 \mathrm{TLA}^{+}$}

$\mathrm{TLA}^{+}$is a formal language for system specification based on Temporal Logic of Actions. It is used to model temporal behavior of software systems. The models are amenable to formal verification by model checking or theorem proving. In the following, we give a brief introduction to $\mathrm{TLA}^{+}$by an example, see Fig. 1. The source code of this example can be downloaded from https://bitbucket. org/gefei/tlaweb-models/.

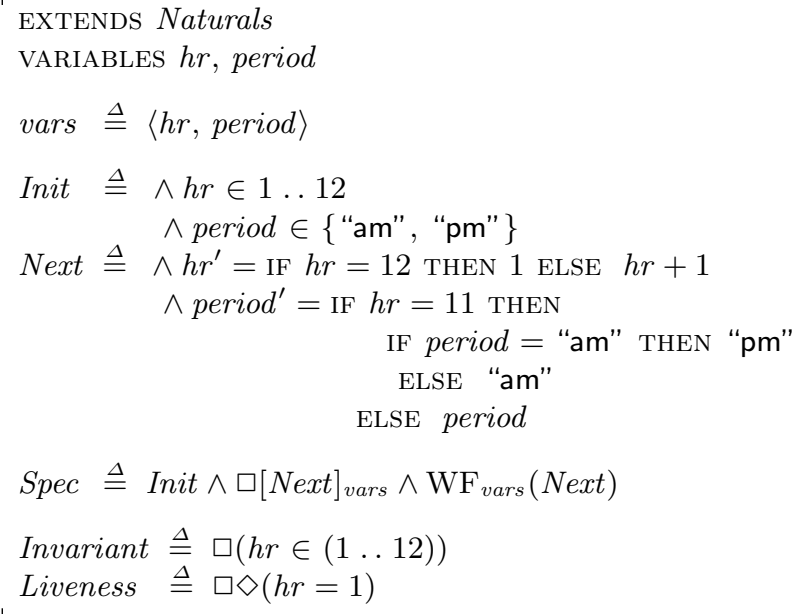

Fig. 1. TLA ${ }^{+}$specification: clock

Our module clock models a 12-hour-clock. It defines two variables to hold the current state of the clock: $h r$ stores the time, and period stores whether it is am or pm. It is common practice in $\mathrm{TLA}^{+}$to define a tuple vars to refer to the variables as a whole. The formula Init defines in the form of conjunction two conditions to be fulfilled in the clock's initial state: $h r$ may be any number between 1 and 12, and period may be either am or pm.

Next is an action. In $\mathrm{TLA}^{+}$, actions define how the system state changes over time, that is, when state change is allowed, and what the succeeding state is. In the clock example, Next does not need any precondition, because the clock is always allowed to proceed to the next hour. The postcondition is defined in prime variables: $h r^{\prime}$ defines the value of $h r$ in the next state, and period' defines the vaule of period in the next state. Our action Next therefore defines declaratively 
how the clock proceeds to he next hour: if the current value of $h r$ is 12 , then its new value is 1 , otherwise it is the current value of $h r$ incremented by 1 ; If (it is $11 \mathrm{am}$ now) the current value of $h r$ is 11 and period is am, then in the next state $(12 \mathrm{pm})$ period should be pm, and if it is $11 \mathrm{pm}$ now, then in the next state period should be am.

Spec is the system specification. It combines the initial state and state transition rules, and states that the rule Next is weakly fair. We ignore the precise definition of fairness; the interested reader is referred to [11]. For now, let it suffice to know by declaring Next as fair, we make sure that the system does not stutter, that is, our clock will not stop.

The temporal operator $\square$ means "always", that is, the succeeding formula is true in every state of the system. With its help, it is easy to define invariants. In our example, the temporal formula Invariant states that it is always true that the value of $h r$ is a number between 1 and 12. The temporal operation $\diamond$ means "eventually", that is, the succeeding formula will be eventually true. The formula Liveness uses a combination of the two operators and states that it is always true that sometimes $h r$ will be 1 . This combination is widely used in temporal logic. A property of the form "it is always true that sometimes something will be true", or "something good will eventually happen", is called a liveness property.

TLC confirms that the two properties of our module are true.

\section{Specifying SPAs with TLA ${ }^{+}$}

Due to its action-based nature, TLA ${ }^{+}$is well-suited to specifying interactive systems like SPAs. We illustrate this by an example. The source code of our model can also be downloaded from https://bitbucket.org/gefei/tlaweb-models/.

Figure 2 shows a simple SPA which provides math exercises to kids. A highlevel design of the GUI is given in Fig. 2(a): the application should display a question (Question) and its running number (Num), wait for the user to input their answer (Answer), and, when the user clicks button Check, show them if the answer is right or wrong (Result) and update a simple statistic of the total numbers of right and wrong answers so far (Count Right and Count Wrong). Furthermore, the user should be able to press button New Question to get a new question presented. Notice that in Fig. 2(a) we use «input» to identify an input field and «button» to identify buttons. These are the widgets where the user can interact with the system. For a better understanding, Fig. 2(b) provides a sample run of the application where the user has just checked their second answer (which is right) and has scored one right and one wrong so far.

Suppose it is required that the user can only click the Check button after they have inputted an answer, and only require a new question after they have

finished (checked) the current one. Further, we suppose the total number of questions is limited.

\subsection{Constants and Variables}

The $\mathrm{TLA}^{+}$module modeling this application is given in Fig. 3. 


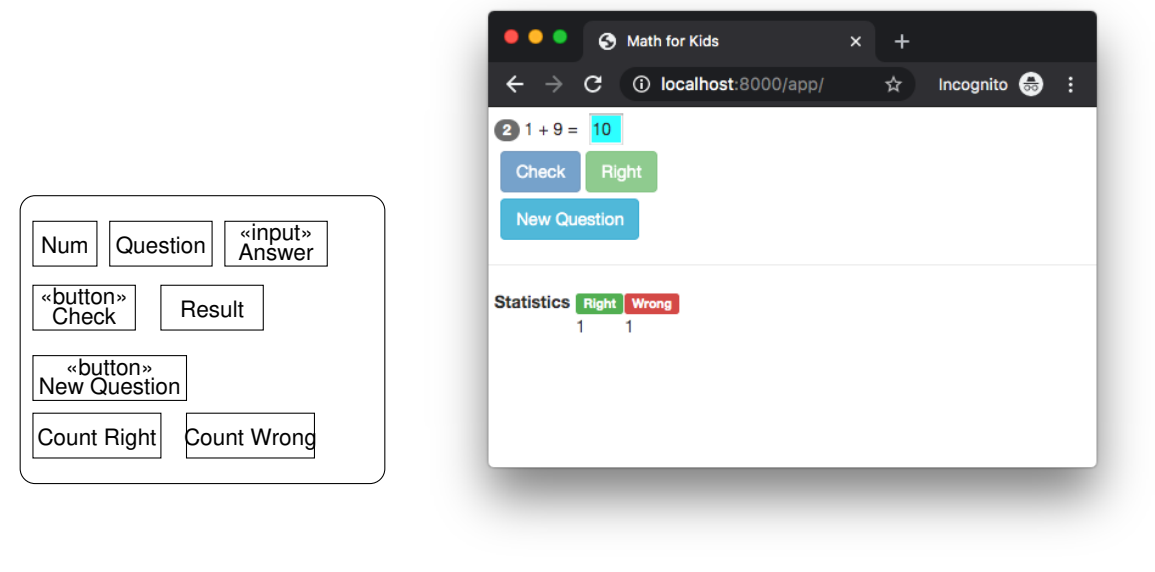

$\begin{array}{ll}\text { (a) Sketch } & \text { (b) Sample run }\end{array}$

Fig. 2. Example: math training

First, we need a constant max_num $q$ to store the maximum number of questions to present to the user. Then we define a variable for each observable property of the widgets:

- we use a variable num to store the number of the current question

- the field for the user to input their answer may be enabled or disabled, we introduce a variable input_enabled to model it,

- the buttons Check and New Question may be either enabled or disabled, we introduce variables check_enabled and new_question_enabled to model them,

- we introduce a variable result to model the result of checking the user's answer to the current question.

- we need two variables count_right and count_wrong to hold the numbers of right and wrong answers so far,

\subsection{Actions}

We define a TLA+ action for each action the user may take. In our sample application, the user may input an answer to the current problem, check if the answer is correct, or get a new problem.

The action Input_Answer models the user action of inputting an answer. The user is only allowed to do this when the input widget is enabled (input_enabled = TRUE). After this action, the only thing we need to change is that the user should be allowed to check their answer now (change_enabled' $=$ TRUE). All other variable should hold their old values.

The action Check models the user action of clicking the button Check and letting the system check if the answer is correct. The only precondition is that 
MODULE math

EXTENDS Naturals

CONSTANT max_num_q

VARIABLE num, count_right, count_wrong, result, input_enabled, check_enabled, new_question_enabled

vars $\triangleq\langle$ num, count_right, count_wrong, result, input_enabled, check_enabled, new_question_enabled $\rangle$

Init $\triangleq \wedge$ num $=1$

$\wedge$ count_right $=0$

$\wedge$ count_wrong $=0$

$\wedge$ input_enabled $=$ TRUE

$\wedge$ check_enabled $=$ FALSE

$\wedge$ new_question_enabled $=$ FALSE

$\wedge$ result $=$ '“'

Input_Answer $\triangleq \wedge$ input_enabled $=$ TRUE

$\wedge$ input_enabled ${ }^{\prime}=$ FALSE

$\wedge$ check_enabled $^{\prime}=$ TRUE

$\wedge$ UNCHANGED $\langle$ num, count_right, count_wrong, new_question_enabled, result $\rangle$

Check $\triangleq \wedge$ check_enabled $=$ TRUE

$\wedge$ check_enabled $^{\prime}=$ FALSE

$\wedge$ new_question_enabled ${ }^{\prime}=$ TRUE

$\wedge \exists$ answer_correct $\in\{$ TRUE, FALSE $\}:$

IF answer_correct $=$ TRUE THEN

$\wedge$ count_right ${ }^{\prime}=$ count_right +1

$\wedge$ result ${ }^{\prime}=$ "Right"

$\wedge$ UNCHANGED count_wrong

ELSE $\wedge$ count_wrong ${ }^{\prime}=$ count_wrong $+1^{\prime}$

$\wedge$ UNCHANGED count_right

$\wedge$ result ${ }^{\prime}=$ "Wrong"

$\wedge$ UNCHANGED $\langle$ num, input_enabled $\rangle$

New_Question $\triangleq \wedge$ num $<$ max_num_q

$\wedge$ new_question_enabled $=$ TRUE

$\wedge$ new_question_enabled $^{\prime}=$ FALSE

$\wedge$ num $^{\prime}=$ num +1

$\wedge$ input_enabled $^{\prime}=$ TRUE

$\wedge$ result ${ }^{\prime}={ }^{\prime \prime \prime}$

$\wedge$ UNCHANGED $\langle$ count_right, count_wrong, check_enabled $\rangle$

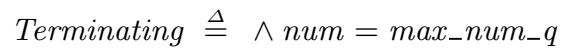

$\wedge$ UNCHANGED vars

Next $\triangleq \quad \vee$ Input_Answer

$\checkmark$ Check

$\vee$ New_Question

$\checkmark$ Terminating

Spec $\triangleq$ Init $\wedge \square[N e x t]_{\text {vars }} \wedge \mathrm{WF}_{\text {vars }}($ Next $)$

Fig. 3. TLA $^{+}$specification: math 
check_enabled must be TRUE. After this action, this variable should be set to FALSE to disable the action. Also, new_question_enabled is set to TRUE to allow the user to get another question. The correctness of the user input is simulated by answer_correct, and the variables result and count_right updated accordingly.

The action New_Question models the user action of getting a new question. The precondition is new_question_enabled $=$ TRUE and num $<$ max_num_ $_{-}$, since we produce at most max_num_q questions. The action sets new_question_enabled to FALSE, and input_enabled to TRUE to allow the user to enter their answer.

The state of the application after initialization is modeled in the action Init. Since the applications shows the user a first question upon initialization, the number of the current question is 1; The user is allowed to input an answer, but not to check the correctness of the answer nor to get a new question yet.

When the maximum number of questions has been reached $\left(\right.$ num $=$ max_num_q $\left._{-}\right)$, then the system is terminated. We model this by the action Terminating, which simply says that the variables do not change any more. Finally, Next comprises the three user actions and Terminating, and Spec defines the overall system behavior. As in the 12-hour clock example, we declare Next to be weakly fair to prevent the system from stuttering.

\section{Model Checking}

Specifying our SPA with TLA ${ }^{+}$allows us to verify some properties of our model formally. This way, $\mathrm{TLA}^{+}$is helpful for finding potential design flaws. In the following we will use TLC, TLA ${ }^{+}$'s model checker, to do this.

Reachability. We first examine the reachability of all questions, that is, for every number $x \in\left\{1 . . m_{a x} n u m_{-} q\right\}$ it holds that sometimes the number of the current question is $x$. This property can be defined as follows:

$$
\text { Reachability } \triangleq \forall x \in 1 \ldots \text { max_num- }_{-}: \diamond(n u m=x)
$$

Running TLC confirms that this property is true.

Liveness. We also examine that if the user is able to input an answer, then sometimes they will also be able to get a new question. In order to express this property, we need the temporal operator $\leadsto$. In $\mathrm{TLA}^{+}$, property $F \leadsto G$ means whenever $F$ is true, then eventually $G$ will be true. In the following, we check that in our module, whenever input_enabled is TRUE, then sometimes new_question_enabled will also be TRUE.

$$
\text { Liveness } \triangleq \text { input_enabled } \leadsto \text { new_question_enabled }
$$

TLC also confirms that this property holds. 
Invariant. Since every answer is checked, it seems obvious that the number of the current question should always equal the sum of the numbers of right and wrong answers so far. We formulate this property as follows:

$$
\text { Invariant } \triangleq \square(\text { num }=\text { count_right }+ \text { count_wrong }) \quad \text { Does not hold! }
$$

However, TLC reports that this property does not hold. The reason is that count_right + count_wrong yields the number of all questions that have been checked, but there may be one question which is presented to the user but not checked yet. Therefore num $_{-} q=$ count_right + count_wrong only holds after the action Check (and before New_Question). That is, it only holds when result is not empty. The following invariant can therefore be verified by TLC:

$$
\text { Invariant } \triangleq \square(\text { result }=\text { '"' } \vee \text { num }=\text { count_right }+ \text { count_wrong }) \quad \text { Correct }
$$

Deadlock. Even applications as simple as the example may be easily erroneous. For example, if we made a "hit-by-one" mistake with New_Question and specified it as

$$
\begin{aligned}
\text { New_Question } \triangleq & \wedge \text { num }<\text { max_num_q }+1 \\
& \wedge \text { new_question_enabled = TRUE } \\
& \wedge \text { new_question_enabled }{ }^{\prime}=\text { FALSE } \\
& \wedge \text { num }^{\prime}=\text { num }+1 \\
& \wedge \text { input_enabled }^{\prime}=\text { TRUE } \\
& \wedge \text { result }=\text { '“'” } \\
& \wedge \text { UNCHANGED }\langle\text { count_right, count_wrong, check_enabled }\rangle
\end{aligned}
$$

then TLC would report a deadlock: In this design, it is possible to do New_Question when num is max_num_q. Then, after this action, num will be incremented to max_num_ $q+1$. Therefore, the precondition of Terminating is violated, the system is deadlocked.

\section{Related Work}

Ever since the emergence of web applications, their formal analysis has been an active research field. Model checking has been recognized as helpful. Research so far mainly focuses on the analysis of explicit navigation structures (hypertextbase applications with explicit links). For an overview, see [1].

Sylvain Hallé et al. [8] propose a method to apply model checking to find potential navigation errors caused by browser functionalities such as bookmarks or "back" button. Deutsch et al. [5] discuss formal verification of WebML [3] models of data-driven web applications using Abstract State Machines $\left(\mathrm{ASM}^{+},[15]\right)$. Miao and Zeng [13] consider a design model and an implementation model of a web application, generate properties from the former, and check with SMV [2] if the latter does has the properties. Knapp and Zhang [9] present a proposal of 
integrating models of UWE [10] into a UML state machine [14] and model check it.

Compared to these methods, our approach uses $\mathrm{TLA}^{+}$as the specification language and its model checker TLC for model verification. More importantly, while the aforementioned methods focus on hypertext-based web applications with explicit navigation structures, our approach aims at documenting and analyzing the workflows of SPAs that are defined implicitly by states of the widgets. In particular, specifying SPAs with $\mathrm{TLA}^{+}$makes it possible to theorem-prove their properties, which will be part of our future work.

\section{Conclusions and Future Work}

We presented a practice friendly and easy-to-use approach to specifying SPA's workflows with TLA ${ }^{+}$. This way, the informal design of SPAs can be documented formally, their implicit workflows make explicit. Our approach helps to understand designs of SPAs better, as well as to find potential flaws in the design.

Future work is possible in several directions. First, we plan to extend our approach to handle more SPA features, such as asynchronous communication or server activities.

Moreover, model checking is only amenable to finite-state systems. We plan to extend your research and to use theorem proving, which is also possible in $\mathrm{TLA}^{+}$. Then it will be possible to analyse infinite-state applications, and we could verify that if we removed the constraint of maximum number of questions, then our math example would run forever.

Last but not least, we plan to extend our research [17] of analysing AngularJS [7] applications to generate the $\mathrm{TLA}^{+}$specification automatically from code. This would greatly improve our abilities to analyse running AngularJS code.

\section{References}

1. Mohammed Yahya Alzahrani. Model Checking Web Applications. PhD thesis, Heriot-Watt University, 2015.

2. Béatrice Bérard, Michel Bidoit, Alain Finkel, François Laroussinie, Antoine Petit, Laure Petrucci, Philippe Schnoebelen, and Pierre McKenzie. Systems and Software Verification, Model-Checking Techniques and Tools, chapter 12. Springer, 2001.

3. Stefano Ceri, Piero Fraternali, Aldo Bongio, Marco Brambilla, Sara Comai, and Maristella Matera. Designing Data-Intensive Web Applications. Morgan Kaufmann, 2002.

4. Edmund M. Clarke, Thomas A. Henzinger, Helmut Veith, and Roderick Bloem, editors. Handbook of Model Checking. Springer, 2018.

5. Alin Deutsch, Liying Sui, and Victor Vianu. Specification and verification of datadriven Web applications. J. Computer and System Sciences, 73(3):442-474, 2007.

6. Jean H. Gallier. Logic for Computer Science: Foundations of Automatic Theorem Proving. Dover Publications, 2018.

7. Google. Angularjs. https://angularjs.org/, 2018. Accessed on 2020-02-01. 
8. Sylvain Hallé, Taylor Ettema, Chris Bunch, and Tevfik Bultan. Eliminating Navigation Errors in Web Applications via Model Checking and Runtime Enforcement of Navigation State Machines. In Charles Pecheur, Jamie Andrews, and Elisabetta Di Nitto, editors, Proc. 25 ${ }^{\text {th }}$ Int. Conf. Automated Software Engineering (ASE'10), pages 235-244. ACM, 2010.

9. Alexander Knapp and Gefei Zhang. Model Transformations for Integrating and Validating Web Application Models. In Heinrich C. Mayr and Ruth Breu, editors, Proc. Modellierung 2006 (MOD'06), volume P-82 of Lect. Notes Informatics, pages 115-128. Gesellschaft für Informatik, 2006.

10. Nora Koch, Alexander Knapp, Gefei Zhang, and Hubert Baumeister. UML-Based Web Engineering: An Approach Based on Standards. In Luis Olsina, Oscar Pastor, Gustavo Rossi, and Daniel Schwabe, editors, Web Engineering: Modelling and Implementing Web Applications, volume 12 of Human-Computer Interaction Series, chapter 7, pages 157-191. Springer-Verlag, 2007.

11. Fred Kröger and Stephan Merz. Temporal Logic and State Systems. Texts in Theoretical Computer Science. An EATCS Series. Springer, 2008.

12. Leslie Lamport. The TLA+Language and Tools for Hardware and Software Engineers. Addison-Wesley, 2003.

13. Huaikou Miao and Hongwei Zeng. Model Checking-based Verification of Web Application. In Proc. 12 $2^{\text {th }}$ Int. Conf. Engineering Complex Computer Systems (ICECCS'07), pages 47-55. IEEE Computer Society, 2007.

14. OMG. Unified Modeling Language, Version 2.5. Specification, Object Management Group, 2015. http://www.omg.org/spec/UML/2.5/PDF/, Accessed on 2020-02-02.

15. Marc Spielmann. Abstract state machines: verification problems and complexity. PhD thesis, Rheinisch-Westfälische Technische Hochschule Aachen, 2000.

16. Wikipedia. Single-page Application. https://en.wikipedia.org/w/index.php? title=Single-page_application\&oldid=938116540, 2020. Accessed: 2020-02-01.

17. Gefei Zhang and Jianjun Zhao. Visualizing Interactions in AngularJS-based Single Page Web Applications. In Proc. 30 ${ }^{\text {th }}$ Int. Conf. Software Engineering 85 Knowledge Engineering (SEKE'18), pages 403-408. KSI Research Inc., 2018. 